(Invited) Second Harmonic Generation: A Powerful Non-Destructive Characterization Technique for Dielectric-on-Semiconductor Interfaces

To cite this article: Irina lonica et al 2020 ECS Trans. 97119

View the article online for updates and enhancements. 


\title{
Second Harmonic Generation: A Powerful Non-Destructive Characterization Technique for Dielectric-on-Semiconductor Interfaces
}

\author{
Irina Ionica $^{\mathrm{a}}$, Dimitrios Damianos ${ }^{\mathrm{a}}$, Anne Kaminski-Cachopo ${ }^{\mathrm{a}}$, Danièle Blanc-Pélissier ${ }^{\mathrm{b}}$, \\ Gerard Ghibaudo $^{\mathrm{a}}$, Sorin Cristoloveanu ${ }^{\mathrm{a}}$, Lionel Bastard ${ }^{\mathrm{a}}$, Aude Bouchard ${ }^{\mathrm{a}}$, Xavier \\ Mescot $^{\mathrm{a}}$, Martine Gri ${ }^{\mathrm{a}}$, Ming Lei ${ }^{\mathrm{c}}$, Brian Larzelere ${ }^{\mathrm{c}}$, and Guy Vitrant ${ }^{\mathrm{a}}$ \\ ${ }^{a}$ Univ. Grenoble Alpes, CNRS, Grenoble-INP, IMEP-LAHC, 38000 Grenoble, France \\ ${ }^{\mathrm{b}}$ INL-UMR 5270, INSA de Lyon, 7 avenue Jean Capelle, 69621 Villeurbanne, France \\ ${ }^{\mathrm{c}}$ FemtoMetrix, 1850 East Saint Andrew Place, Santa Ana, California 92705, USA.
}

The second harmonic generation ( $\mathrm{SHG}$ ) proved to be a very promising characterization technique for dielectric-semiconductor interfaces because it is sensitive, non-destructive, can be applied directly on wafer, at different stages of wafer processing. The method, based on non-linear optics effects, is measuring a signal encompassing the "static" electric field at the dielectricsemiconductor interface which is directly related to the oxide charges $Q_{o x}$ and to the interface state density $D_{i t}$. A general methodology for $\mathrm{Q}_{\mathrm{ox}}$ and $\mathrm{D}_{\text {it }}$ extraction from SHG measurements requires (i) calibration based on parameters obtained by classical electrical methods and (ii) modeling to capture the optical propagation phenomena that impact the SHG signal. In this paper, we discuss these issues based on a review of our recent advances on how to exploit SHG for dielectrics on semiconductor characterization.

\section{Introduction}

The dielectric-on-semiconductor stacks are omnipresent in devices for many application fields such as micro-nano electronics, photovoltaics (1), image sensors (2), bio-chemical sensors, etc. In each case, the electrical quality of the interface has a tremendous impact on the performances of the device. Two parameters are generally used to determine the electrical quality of such an interface: the fixed oxide charge density $\mathrm{Q}_{\text {ox }}$ and the interface state density $D_{i t}$. Most of the times, these parameters are accessed through electrical measurements, such as current, capacitance, noise (3), followed by adapted extraction methods and implemented on specially fabricated test-devices (e.g.: metal-oxidesemiconductor - MOS capacitances or transistors). Some other methods can be directly implemented at the wafer-level, without any additional fabrication steps for a test device, e.g.: Corona-Kelvin characterization of semiconductors (4), carrier lifetime extraction (5) conducted through photoconductance or photoluminescence decay measurements. Besides the possibility of direct on-wafer probing without any addition steps, the criteria to choose the most adapted measurement method also involves sensitivity, nondestructiveness, ability to discriminate $D_{i t}$ and $Q_{o x}$, capability to provide high spatial resolution. A recent technique which could meet all these criteria is the Second Harmonic Generation (SHG) (6), based on non-linear optics effects. 


\section{$\underline{\text { SHG - principle }}$}

The SHG is a non-linear optics effect, associated with the second order non-linear polarization $\vec{P}^{(2)}$ of a material, under the illumination with a high-intensity laser with angular frequency $\omega$, wavelength $\lambda$ and electric field amplitude $E(\omega)(7)$ :

$$
\vec{P}^{(2)}(2 \omega)=\vec{\chi}^{(2)} \cdot \vec{E}(\omega) \cdot \vec{E}(\omega)
$$

where $\vec{\chi}^{(2)}$ is the second order susceptibility tensor of the material.

In dipolar approximation, the centrosymmetric materials do not exhibit bulk secondorder polarization because of the crystal symmetry. Consequently, materials such as silicon, amorphous $\mathrm{SiO}_{2}$, alumina, $\mathrm{HfO}_{2}$ and other high-k dielectrics have no bulk dipolar susceptibility. However, the centro-symmetry is broken at the interface between two different materials which will generate SHG and open the possibility to obtain specific information from interfaces when measuring the generated SHG signal from a whole structure. In particular the magnitude of the SHG signal will depend on the properties of interfaces that can be described through an "effective" nonlinearity as:

$$
\vec{P}^{(2)}(2 \omega)=\vec{\chi}_{\text {interface }}{ }^{(2)} \cdot \vec{E}(\omega) \cdot \vec{E}(\omega)
$$

Nevertheless, this is a simple view and several other SHG sources will be added to pure interface contributions. They will be included in the detected overall SH signal generated by the whole structure. This is the case with EFISH (Electric Fields Induced Second Harmonic). All materials, including of course centro-symmetric ones, exhibit a third order non-linearity described by the susceptibility tensor $\ddot{\chi}^{(3)}$. In particular it is well known that strong static electric fields are present at the vicinity of interfaces between silicon and dielectrics. They will generate SHG though $\vec{\chi}^{(3)}$ and we obtain:

$$
\vec{P}^{(2)}(2 \omega)=\left(\vec{\chi}_{\text {interface }}{ }^{(2)}+\vec{\chi}^{(3)} \cdot E_{D C}\right) \cdot \vec{E}(\omega) \cdot \vec{E}(\omega)
$$

Just note that by DC we consider slowly varying electric fields at frequencies far from optical frequencies, which therefore can extend to several tens of $\mathrm{GHz}$ and far more. Other phenomena at the origin of SHG signal are present in addition to these two ones. This is the case for anisotropic nonlocal bulk contribution. If amorphous materials can be considered as everywhere centrosymmetric on average view, this is not the case for crystalline materials. Due to their organized atomic structure, a non-zero SHG contribution emerges from bulk materials, as pointed out in pioneering works by Sipe et al (8). However this effect is very weak and has been ignored in most articles and textbooks but with the development of femtosecond lasers and their use as nonlinear probes (as is the case in this paper), we are looking at weak SHG signals and it has to be considered. At the end, the intensity emitted at the double frequency, $I^{2 \omega}$ is given by the square modulus of the polarization:

$$
I^{2 \omega} \sim\left|\vec{\chi}_{\text {interface }}{ }^{(2)}+\vec{\chi}^{(3)} \cdot \vec{E}_{D C}\right|^{2} \cdot\left|I^{\omega}\right|^{2}
$$


At interfaces between dielectrics and semiconductors, the "static" electric field that could be extracted from the SHG intensity, can have two terms: one associated to the fixed charges $\mathrm{Q}_{\mathrm{ox}}$ and the initially charged traps $\left(\mathrm{E}_{0}\right)$ and the second one due to trapping/detrapping effects related to $\mathrm{D}_{\mathrm{it}}$, that can vary in time, $\mathrm{E}(\mathrm{t})$. In this case, the SHG gives access to $\mathrm{Q}_{\mathrm{ox}}$ and $\mathrm{D}_{\mathrm{it}}$ and its intensity writes as:

$$
I^{2 \omega} \sim\left|\vec{\chi}_{\text {interface }}{ }^{(2)}+\vec{\chi}^{(3)} \cdot\left(\vec{E}_{0}+\vec{E}(t)\right)\right|^{2} \cdot\left|I^{\omega}\right|^{2}
$$

Both fundamental and second harmonic signal are propagating waves at optical frequencies. Optical electric field intensities will depend on propagation in the whole stratified structure. It is crucial to precisely describe these propagation effects, including absorption and multiple interferences phenomena. This is achieved by rigorously solving Maxwell equations without any approximation at both fundamental and $\mathrm{SH}$ frequencies. In particular all boundary electromagnetic conditions are rigorously satisfied with vectorial fields for all polarizations. Nonlinear polarization at interfaces should be included as a source term in Maxwell equations at SH frequency. We noticed that rigorous modeling of propagation effects is crucial since small variations of layer thicknesses as small as some nanometers can affect the magnitudes of optical fields significantly. This will induce variations of overall detected SHG signal by several tens of \% which cannot be ignored and has to be precisely described and included in models though propagation at both frequencies as we did in this work.

The problem is even more complex for multiple interfaces, because the second order polarization from equation [3] appears at each one of them and the corresponding emitted SH waves are traveling in the sample and interfere together. If additionally the sample under study contains multiple interfaces which are electrically coupled (that is the case in thin silicon-on-insulator substrates), the electric fields to be considered for each interface are not independent of each other (9), adding a supplementary degree of complexity. Before moving deeper into those aspects, we present a state-of-art characterization based on SHG.

\section{$\underline{\text { SHG for characterization of interfaces - state of art }}$}

A typical experimental setup of SHG can allow variation of multiple parameters such as the incident wavelength $\lambda$, incident power, input/output polarization, angle of incidence, azimuthal angle of the sample (Figure 1). The SHG measured versus each one of these parameters provides different type of information on the sample.

For example, the spectroscopic SHG (SHG versus $\lambda$ ) allows to identify inter-band resonances at silicon interfaces, as well as the different band contributions (10). Various input/output polarization configurations were used to determine specific components of the susceptibility tensor (11), (12), (13). The SHG variation with the azimuthal angle of the sample (rotational angle around the normal to its surface) provided the substrate symmetry (14) or was related to the surface roughness (15). 


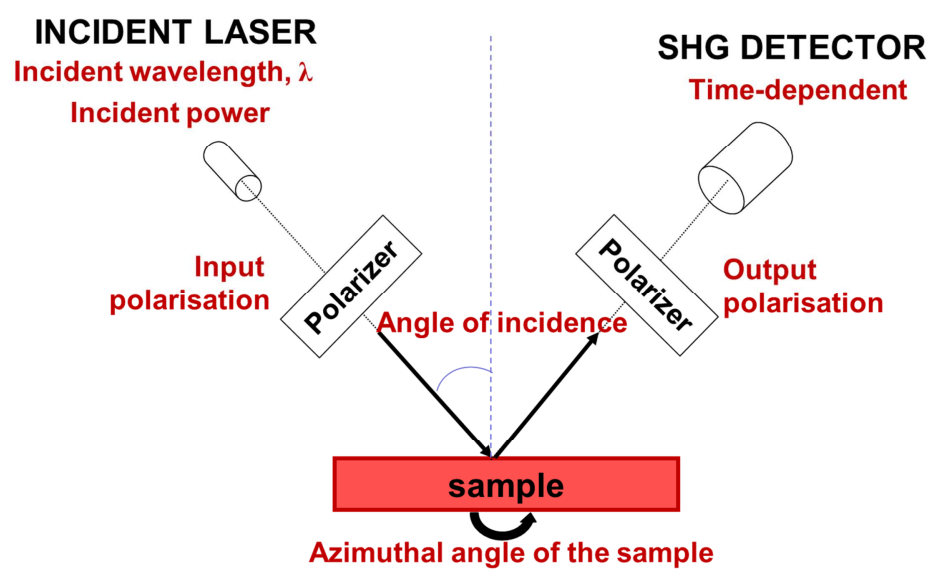

Figure 1. Schematic of the experimental SHG configuration, with the key parameters.

As seen from equation [5], SHG variation with time relates to trapping/de-trapping phenomena; the method was used for a variety of dielectric materials: $\mathrm{SiO}_{2},(6),(16)$, (17) $\mathrm{Al}_{2} \mathrm{O}_{3}(18), \mathrm{ZrO}_{2}$ etc. In this kind of studies, the carrier injection, which depends on the incident power, is related to multi-photon excitation processes activated during the SHG measurements. For instance in oxidized silicon highly doped with boron, the power variation allowed to identify contributions from boron-induced defects and oxygen traps on the top oxide surface (16). Among the high-k dielectric materials, alumina $\mathrm{Al}_{2} \mathrm{O}_{3}$ is one of the mostly studied with SHG. Spectroscopic (18), azimuthal (19), time-dependent SHG were used by different groups to study the polarity of the charges contained in alumina, eventually activated by annealing, or their change in sign for different intermediate $\mathrm{SiO}_{2}$ layer thicknesses.

The sensitivity of the technique in all these cases is high, however having a more general approach with a calibration procedure, would allow a wider extension of the use of the SHG for interface characterization. In order reach this aim, in this paper we present some of the issues of the SHG from single and multiple interface structures, using both experimental and simulated data and focusing on the impact of optical phenomena on the SHG.

\section{SHG: Experimental setup and simulations}

The measurements were performed on a commercial tool from FemtoMetrix (20), with a pump laser of $780 \mathrm{~nm}$ wavelength emitting femtosecond pulses with $80 \mathrm{MHz}$ repetition rate, $95 \mathrm{fs}$ pulse duration and an average power of $360 \mathrm{~mW}$. The incident beam polarization is controlled by a half wave plate. The SHG can be measured at particular output polarization angles selected by a rotating polarizer. For most of the experiments we used p polarizations for both input and output (laser electric field and SHG electric field are parallel to the plane of incidence). The SH generated from the sample is properly filtered from the reflected fundamental light and the photons are detected by a photomultiplier coupled with a gated photon counter. The layer thickness can be measured at the same spots as the SHG, with a reflectometer integrated in the tool.

Being based on optics, the SHG is strongly impacted by optical phenomena such as absorption, transmission, and interferences, on both the fundamental and the second 
harmonic waves. This is one of the reasons why having a generalized procedure is not obvious and the results in the literature are difficult to compare to one another. However, the optical phenomena can be simulated and a comparison with the experimental measured SHG should lead to a decorrelation between the electrical properties at the interface and the optical interferences in the structure. In order to reach this aim, we developed an "in-house" simulator for the SHG calculation in multi-layer structures. Based on the matrix formalism [40], our simulation tool estimates the optical electric field at the fundamental and $\mathrm{SH}$ frequency at all the interfaces in the multilayer. The second order polarization was calculated at each interface using the typical expressions for $\mathrm{Si}\left(\begin{array}{lll}1 & 0 & 0\end{array}\right)(8)$ and was then added as a source term in the Maxwell's equations at that particular interface. For some simulations, we added a "static" interface electric field corresponding to the EFISH, by modifying the appropriate susceptibility component. The fields at the $2^{\text {nd }}$ harmonics locally generated at every interface are obtained from the boundary conditions and are then propagated through the whole sample. The SHG intensity coming out the structure is calculated as the square modulus of the SH field in the air, above the sample. More details are given elsewhere (21).

In the next sections, we use both experimental and simulation results to show the main problematics when using SHG for $\mathrm{Q}_{\mathrm{ox}} / \mathrm{D}_{\mathrm{it}}$ extraction from multi-layered structures.

\section{SHG for single dielectric-semiconductor interfaces}

Our first study case is a structure with a single dielectric-semiconductor interface obtained by deposition of alumina $\left(\mathrm{Al}_{2} \mathrm{O}_{3}\right)$ on p-type silicon. The reasons of choosing alumina are multiple:

- alumina is widely used for passivation of silicon for solar cells (22), and image sensors (23);

- alumina is known to have fixed charges $\mathrm{Q}_{\mathrm{ox}}$ attributed to $\mathrm{Al}$ vacancies and interstitial $O$, that are used for the so called field-effect passivation (24). Depending on the fabrication steps (i.e. annealing) these fixed charges can be activated or not;

- depending on the deposition technique, alumina with different $D_{\text {it }}$ values can be produced. Consequently with this kind of samples, one should be able to separate the impact of $\mathrm{Q}_{\mathrm{ox}}$ and the one of $\mathrm{D}_{\mathrm{it}}$.

For this study, we chose 4 samples with different $Q_{o x} / D_{i t}$, fabricated by thermal or plasma-enhanced atomic layer deposition (ALD), annealed or not, as in (25). Complementary characterization of samples using capacitance versus voltage measurements (21) allowed to extract $\mathrm{Q}_{\mathrm{ox}}$ and $\mathrm{D}_{\mathrm{it}}$ (see TABLE I).

TABLE I. $\mathrm{Al}_{2} \mathrm{O}_{3}$ on silicon samples and their $\mathrm{Q}_{\mathrm{ox}} / \mathrm{D}_{\mathrm{it}}$ parameters extracted from capacitance measurements. T0, T1 and P0, P1 were deposited by thermal and plasma-enhanced ALD respectively. T0, $\mathrm{P} 0$ were measured as-deposited while T1, P1 were annealed at $400^{\circ} \mathrm{C}$ for 10 minutes.

\begin{tabular}{ccc}
\hline Sample & $\mathbf{Q}_{\mathbf{o x}}\left(\mathbf{1 0}^{\mathbf{1 2}} \mathbf{c m}^{\mathbf{- 2}}\right)$ & $\mathbf{D}_{\mathbf{i t}}\left(\mathbf{e V}^{\mathbf{- 1}} \mathbf{c m}^{\mathbf{- 2}}\right)$ \\
\hline T0 & +2.85 & $<10^{10}$ \\
\hline T1 & -0.5 & $<10^{10}$ \\
\hline P0 & -2.64 & $10^{13}$ \\
\hline P1 & -3.58 & $3 \times 10^{12}$ \\
\hline
\end{tabular}


Figure 2(a) shows the time-dependent SHG measured from the four samples. The signal was recorded for more than 20 minutes for each sample. The behavior of the curves is similar: after the first "fast" increase, the SHG signal saturates. This typical time-dependent SHG corresponds to samples in which an initial interface electric field is modified by the charges generated and injected from the semiconductor into the oxide layer during the measurement (Figure 2b). An obvious increase in the SHG signal is observed for the annealed samples which are known to have a higher concentration of negative charges $\left(\mathrm{Q}_{\mathrm{ox}}\right)$; this is consistent with a higher EFISH signal due to larger interface field. The time evolution of the SHG at the beginning of the measurement is slower for the samples with a lower $\mathrm{D}_{\text {it }}$ level, which is in agreement with a lower level of charge trapping for these cases, associated with a smaller EFISH.

(a)

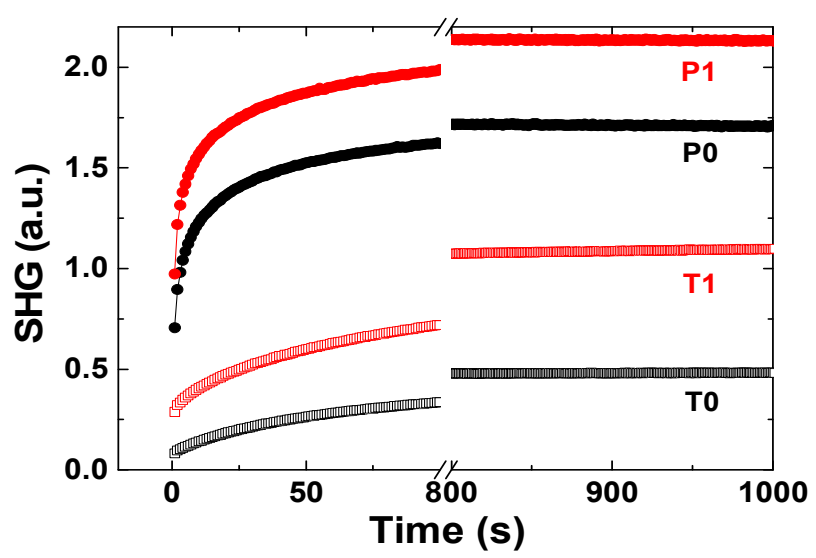

(b)

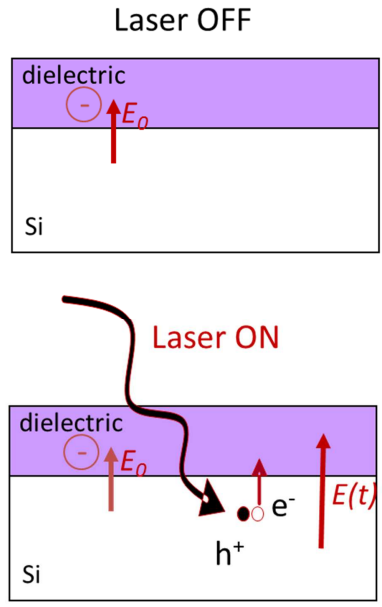

Figure 2. (a) SHG signal versus time measured for four alumina samples with the $\mathrm{Q}_{\mathrm{ox}}, \mathrm{D}_{\text {it }}$ values given in Table I. The time scale is broken in order to show both the zoom at the beginning of the measurement and the saturation response. The measurements were performed at $45^{\circ}$ incidence angle, for $\mathrm{P}$-input/P-output polarizations. (b) Interface electric field in the sample is modified by the charges generated and injected during the SHG measurement.

According to equation [5], the initial SHG point (at $\mathrm{t}=0 \mathrm{~s}$ ) is directly related to the square of the initial static field $\left(\mathrm{E}_{0}\right)$ in the structure that can be calculated using Gauss equation: $E_{0}=Q_{o x} / \varepsilon_{S i}$, where $\varepsilon_{S i}$ is the dielectric permittivity of silicon. Calculated $\mathrm{E}_{0}$ values for the four samples are reported on Figure 3 which shows the square root of the SHG intensity versus the initial electric field. The linear dependence expected from equation [5] is fairly well respected, meaning that this kind of curve could be used as calibration: the initial SHG measurement on a new sample, would give $E_{0}$ that could be used to simply calculate the oxide charge $Q_{\text {ox }}$. Even though very tempting, this approach would not be correct because the response of the SHG is also related to the geometry and optical properties of the samples. In the case of alumina, the dielectric layer is transparent for both fundamental and SHG, but its thickness changes the optical path and therefore the SHG signal. 


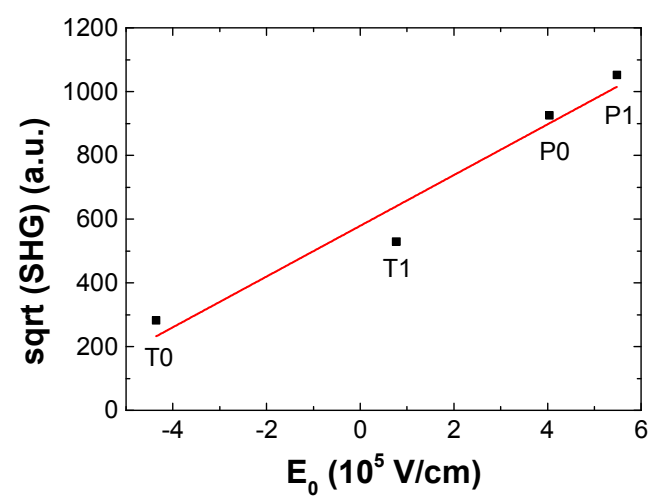

Figure 3. Square root of the initial SHG values from Figure 2 represented versus the static electric field calculated with the fixed oxide charges of each sample from Table I.

In Figure 4 SHG was measured on three samples of different thicknesses 5, 15 and 25 $\mathrm{nm}$ before and after annealing that activates Qox. SHG was also calculated for these structures, using our in-home simulator described in the previous section. The simulated response before annealing takes into account only the optical effects. For the simulation corresponding to the samples after annealing, an electric field of $10^{5} \mathrm{~V} / \mathrm{cm}$ was added at the interface. The normalized SHG simulated data reproduce the experimental thickness dependence trend of the normalized measured SHG signal. The activation of the negative charges in the alumina layer gives rise to higher EFISH in both measurements and simulations, for each thickness.

As seen in all these examples, the SHG evidences very well the field effect passivation but interpreting SHG response must take into account the optical effects even in samples with only one interface and with a dielectric layer that is transparent. Strategies for the results to be unaffected by the effect of the propagation phenomena must be implemented. A possible solution might be the use of SHG measurement versus another parameter (e.g. the angle of incidence, as in (21)) or to use simulations with and without EFISH in order to estimate the impact of the optical effects and normalize the data with respect to the optical effects.

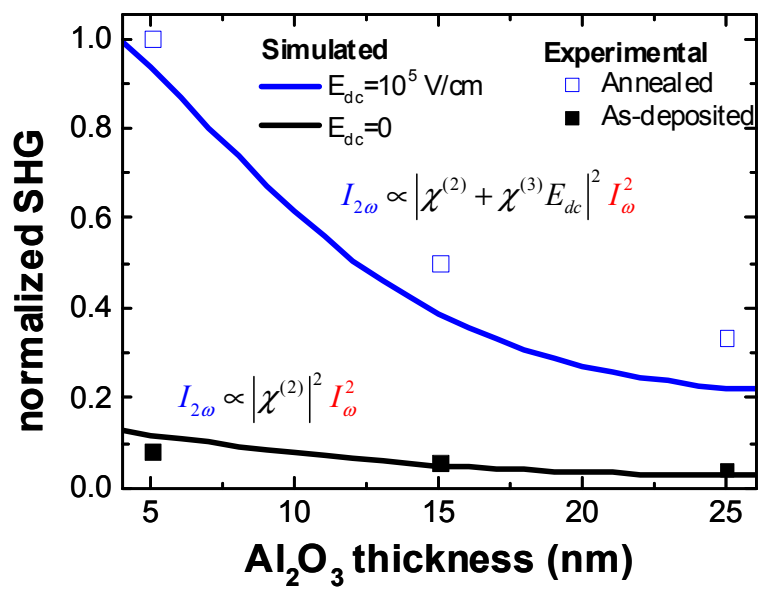

Figure 4. Experimental and simulated SHG intensity versus $\mathrm{Al}_{2} \mathrm{O}_{3}$ thickness. The measurements were performed at $45^{\circ}$ angle for $\mathrm{P}$-input/P-output polarizations. The normalization was done by dividing each set of values (experimental and simulated) with its corresponding maximum value. 


\section{SHG for multiple dielectric-semiconductor interfaces}

The study of a multilayer structure implicates including additional concerns:

- the second harmonic is potentially generated at all the interfaces;

- depending on the layers, some might be absorbing the fundamental and/or SHG signals.

A nice study-case to show these effects is silicon-on-insulator (SOI). Indeed, in SOI, the second harmonic can be generated at 3 interfaces: (1) bulk silicon - buried oxide (BOX), (2) BOX - silicon film and (3) Si film - top oxide (native or passivation oxide). Additionally for thin films SOI, the two interfaces of the film can be coupled from the electrical point of view, which will add more complexity to the analysis.

However an implementation of the SHG for SOI characterization would be very advantageous since the methods typically used to extract electrical properties of SOI wafers (i.e. interface trap densities) necessitate either full fabrication of test devices (which is not time and cost-effective) or placing metallic probes on the film (highly destructive especially for ultra-thin films) (26). Within this context, the non-destructive SHG, sensitive to interface electric fields has a great potential. Previous studies of SOI by SHG evidenced charging of the buried oxide due to radiation (27), metal contamination on the top of the silicon film (28) or even reproduced pseudo-MOSFET characteristics (27).

When dealing with SOI, the interface that needs characterization is the buried one, between the film and the BOX. The second harmonic generated at $390 \mathrm{~nm}$ must then travel through the silicon film and be transmitted outside the sample, towards the detection stage. The absorption length in the silicon film, at the second harmonic is of about $70 \mathrm{~nm}$ (and of about $10 \mu \mathrm{m}$ at the fundamental). Consequently for the Si films thicker than the 70 $\mathrm{nm}$, all the signal of SHG detected would come from the top interface ( $\mathrm{Si}$ - passivation oxide). Nevertheless, the geometry of the film and BOX influence the interference pattern at the fundamental, so even the SHG generated at the top interface might be modified.

In order to evidence this, we measured SHG and the film thickness at the same spots, on two wafers having $145 \mathrm{~nm} / 1000 \mathrm{~nm}$ and $88 \mathrm{~nm} / 145 \mathrm{~nm}$ film/BOX thicknesses respectively (insets of the Figure $5 \mathrm{a}$ and b). The SHG data was then represented versus the film thickness. We simulated the structures, without any interface electric fields. Figure 5 shows the experimental normalized SHG as points and the simulated SHG as lines. For both wafers the simulation without any static electric field reproduces quite well the experimental data. Note that in both cases the interface that gives most of the SHG is the top one, but the field at the fundamental frequency travels different paths and its local value at the top interface will be different, and so will be the SHG. 
(a)

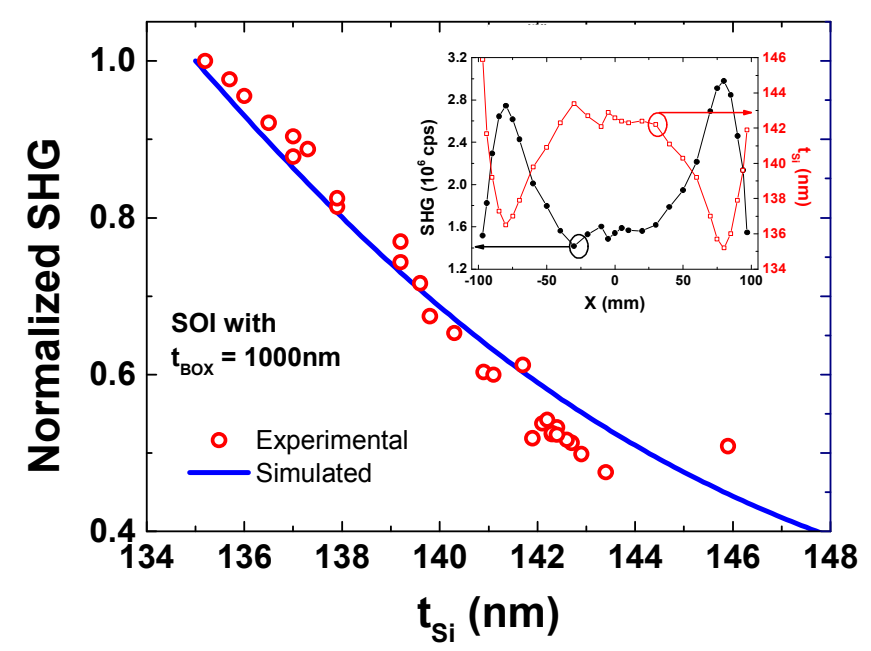

(b)

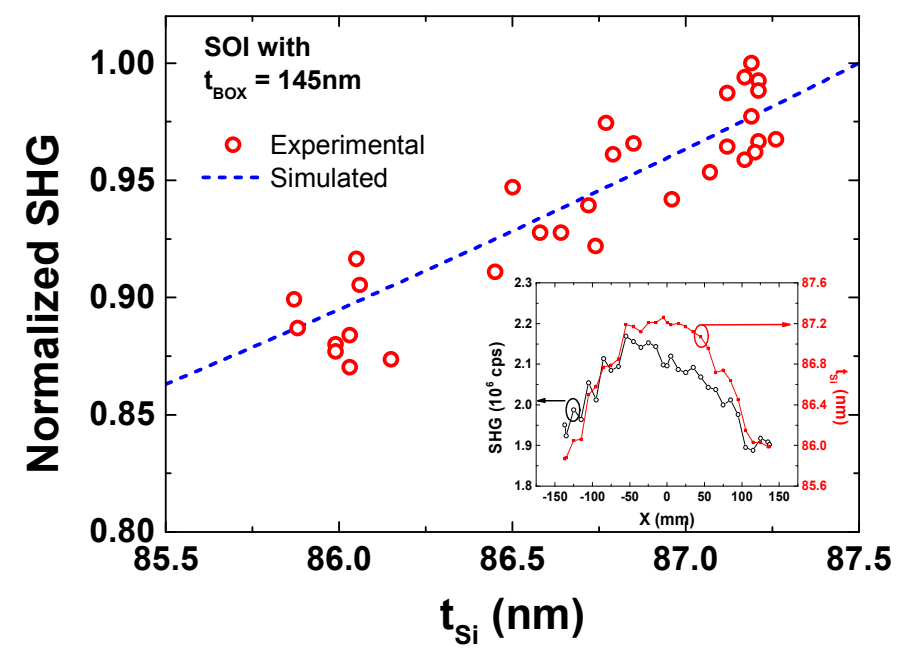

Figure 5. Normalized SHG versus Si film thickness for thick (a) and thin (b) SOI: model (lines) and experiment (data points). The normalized experimental data points were calculated from the corresponding graphs inserted. The normalization was done by dividing each set (experimental and simulated) by its corresponding maximum value. SHG and Si film thickness measured on the same spots on thick (a) and thin (b) SOI wafers. The $\mathrm{X}$-axis represents different measurement spots on each wafer, across its diameter. The angle of incidence was set at $45^{\circ}$ and input/output polarizations are $\mathrm{P} / \mathrm{P}$.

The fundamental field is also modified with the angle of incidence that affects its optical path inside the structure. Tracing the SHG versus the angle of incidence (AOI) is another way to validate the effect of the geometry on both experiments and simulations. Figure 6 shows normalized SHG versus AOI for two SOI geometries, $88 \mathrm{~nm}$ film $/ 145 \mathrm{~nm}$ BOX and $24 \mathrm{~nm}$ film $/ 25 \mathrm{~nm}$ BOX. For the thicker SOI, the simulation without any interface field reproduces quite well the experiments. Adding a static field doesn't modify the signal, since it is the top interface that dominates in this case. For the thinner SOI the experimental SHG data and the simulation with no electric field are shifted by more than $20^{\circ}$. Optical phenomena alone are not sufficient to reproduce the experimental results, but adding an electric field $\mathrm{E}_{\mathrm{DC}}\left(10^{4} \mathrm{~V} / \mathrm{cm}\right.$ and $\left.10^{5} \mathrm{~V} / \mathrm{cm}\right)$ at the interface approaches the simulations to the measured data. The higher electric field is a better fit since in thin SOI the $\mathrm{Si} / \mathrm{SiO}_{2}$ interfaces are electrically coupled together and the electric field can be strong (9). Therefore when thin Si films are used, SHG measurements allow accessing the interfacial electric field partially due to the interface trap density. 
$88 \mathrm{~nm} / 145 \mathrm{~nm}$ SOI wafer

(a)

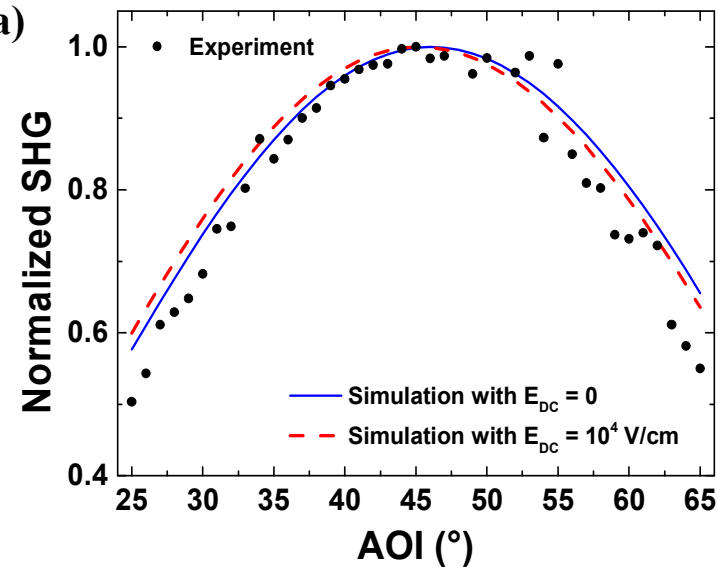

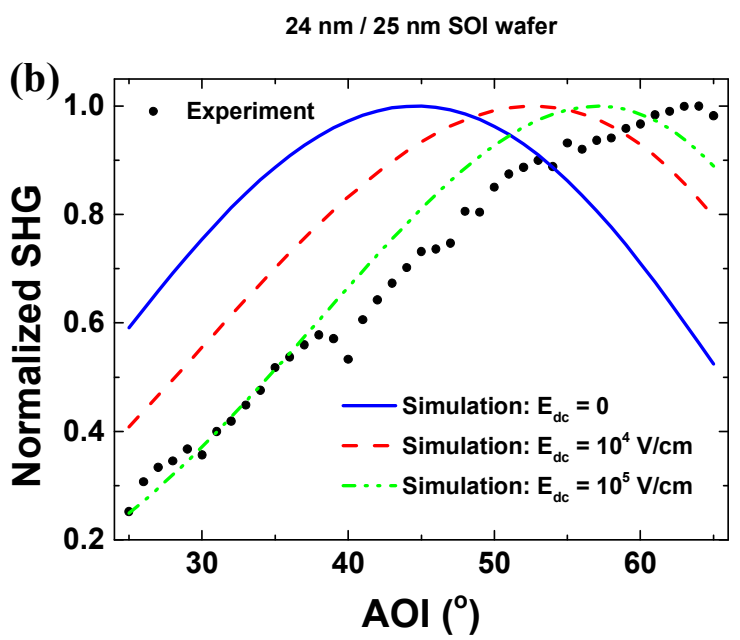

Figure 6. (a) Normalized SHG versus angle of incidence in SOI with $88 \mathrm{~nm}$ Si film thickness and $145 \mathrm{~nm}$ BOX thickness: model (lines) and experiment (data points). A simulation with a field of $10^{4} \mathrm{~V} / \mathrm{cm}$ was added. (b) Normalized SHG versus angle of incidence in SOI with $24 \mathrm{~nm}$ Si film thickness and $25 \mathrm{~nm}$ BOX thickness: model (lines) and experiment (data points). For the modeling, three different values of $\mathrm{E}_{\mathrm{DC}}$ were tested: $0,10^{4} \mathrm{~V} / \mathrm{cm}$ and $10^{5} \mathrm{~V} / \mathrm{cm}$.

Note that the interface of interest is the film-BOX, while the one that dominates the SHG is the top one (film-passivation or native oxide) (29). However if the SOI film thickness is below the absorption limit at the double frequency ( $70 \mathrm{~nm}$ thickness for 380 $\mathrm{nm}$ wavelength of SHG), it is possible to develop a strategy to access the buried interface. In order to confirm this, we simulated the SHG response of an SOI structure with $12 \mathrm{~nm}$ film and $145 \mathrm{~nm}$ BOX thicknesses for various electric fields at the interface film-BOX, $\mathrm{E}_{2}$ (Figure 7a). Additionally, two different values of top-interface fields were considered. Figure $7 \mathrm{~b}$ shows the linear dependence of the square root of SHG intensity versus $\mathrm{E}_{2}$, as predicted by equation [5]. Even though the top interface has a much higher impact, the film-BOX is still present and clearly affects the SHG intensity.

(a)

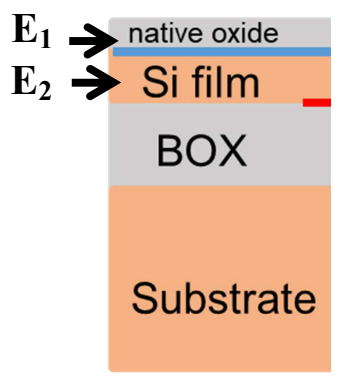

(b)

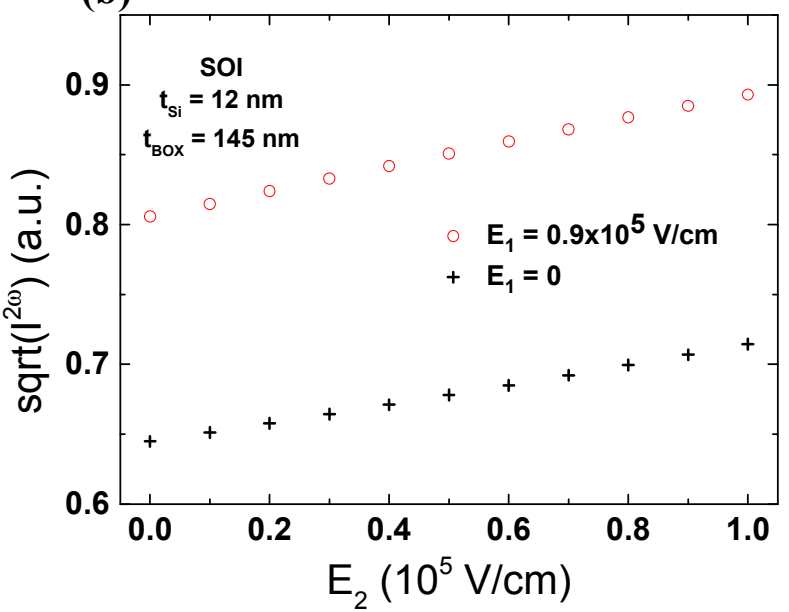

Figure 7. (a) Simulated SOI structure showing the interface between the Si film and the $\mathrm{BOX}$, where the electric fields $\mathrm{E}_{1} / \mathrm{E}_{2}$ were added at the interfaces with the film. (b) Square root of the SHG versus the electric field at the film-BOX interface. 


\section{Conclusion}

The SHG is an optical method that is very sensitive to electrical fields at the interfaces between dielectrics and semiconductors, which are related to $Q_{o x}$ and $D_{i t}$. The method is obviously non-destructive, very well adapted for thin films and it can be applied directly on wafers, without any particular test-structure fabrication. For quantitative evaluations of $\mathrm{Q}_{\mathrm{ox}}$ and $\mathrm{D}_{\mathrm{it}}$ through stand-alone $\mathrm{SHG}$, the optical phenomena that depend on the sample geometry need to be correctly understood and de-correlated from the electrical properties of the samples. For example, even transparent layers can modify the interference pattern, so the source terms generating second harmonic at the interfaces are modified. Depending on the optical properties of multilayers, the most contributing interface might be the one on the top. However this is not a limitation for thin films and a correct understanding of the optical phenomena allow developing adapted measurement strategies and calibrations in order to extract interface electric fields from SHG measurements.

\section{Acknowledgments}

This work was supported by Region Rhône Alpes (ARC6 program), the French National Research Agency within the framework of the OXYGENE project (ANR-17CE05-0034) and French National Plan Nano2022, within the IPCEI Nanoelectronics for Europe program.

\section{References}

1. A. G. Aberle, Progress in Photovoltaics: Research and Applications, 8(5), (2000).

2. J. L. Regolini, D. Benoit, and P. Morin, Microelectronics Reliability, 47(4), (2007).

3. D. K. Schroder, Semiconductor Material and Device Chracterization, John Wiley \& sons, New Jersey, (2006)

4. M. Wilson, J. Lagowski, L. Jastrzebski, A. Savtchouk, and V. Faifer, AIP Conference Proceedings, 550(1), (2001).

5. D. K. Schroder, Electron Devices, IEEE Transactions on, 44(1), (1997).

6. G. Lupke, Surface Science Reports, 35(35), (1999).

7. P. N. Butcher and D. Cotter, The Elements of Nonlinear Optics, Cambridge University Press, (1991)

8. J. E. Sipe, D. J. Moss, and H. M. van Driel, Physical Review B, 35(3), (1987).

9. G. Hamaide, F. Allibert, F. Andrieu, K. Romanjek, and S. Cristoloveanu, SolidState Electronics, 57(1), (2011).

10. W. Daum, Applied Physics A, 87(3), (2007).

11. K. Kotaro, T. Hideo, and F. Atsuo, Japanese Journal of Applied Physics, 30(5R), (1991).

12. X. Li, J. Willits, S. T. Cundiff, I. M. P. Aarts, A. A. E. Stevens, and D. S. Dessau, App Phys Lett, 89(2), (2006).

13. J. Sipe, D. Moss, and H. Van Driel, Physical Review B, 35(3), (1987).

14. H. W. K. Tom, T. F. Heinz, and Y. R. Shen, Phys Rev Lett, 51(21), (1983). 
15. J. I. Dadap, B. Doris, Q. Deng, M. C. Downer, J. K. Lowell, and A. C. Diebold, App Phys Lett, 64(16), (1994).

16. H. Park, J. Qi, Y. Xu, K. Varga, S. M. Weiss, B. R. Rogers, G. Lupke, and N. Tolk, phys. stat. sol. (b), 247(8), (2010).

17. B. Jun, Y. V. White, R. D. Schrimpf, D. M. Fleetwood, F. Brunier, N. Bresson, S. Cristoloveanu, and N. H. Tolk, App Phys Lett, 85(15), (2004).

18. J. J. H. Gielis, B. Hoex, M. C. M. van de Sanden, and W. M. M. Kessels, J App Phys, 104(7), (2008).

19. N. M. Terlinden, G. Dingemans, V. Vandalon, R. H. E. C. Bosch, and W. M. M. Kessels, J App Phys, 115(3), (2014).

20. http://femtometrix.com,

21. D. Damianos, G. Vitrant, A. Kaminski-Cachopo, D. Blanc-Pelissier, G. Ghibaudo, M. Lei, J. Changala, A. Bouchard, X. Mescot, M. Gri, S. Cristoloveanu, and I. Ionica, J App Phys, 124(12), (2018).

22. S. W. Glunz, J. Benick, D. Biro, M. Bivour, M. Hermle, D. Pysch, M. Rauer, C. Reichel, A. Richter, M. Rüdiger, C. Schmiga, D. Suwito, A. Wolf, and R. Preu, 2010 35th IEEE Photovoltaic Specialists Conference, (2010).

23. J. P. Carrère, S. Place, J. P. Oddou, D. Benoit, and F. Roy, 2014 IEEE International Reliability Physics Symposium, (2014).

24. G. Dingemans and W. M. M. Kessels, Journal of Vacuum Science \& Technology $A, \mathbf{3 0}(4)$, (2012).

25. I. Ionica, D. Damianos, A. Kaminski, G. Vitrant, D. Blanc-Pélissier, J. Changala, M. Kryger, C. Barbos, and S. Cristoloveanu, ECS Transactions, 72(2), (2016).

26. S. Cristoloveanu, M. Bawedin, and I. Ionica, Solid-State Electronics, 117, (2016).

27. B. Jun, R. D. Schrimpf, D. M. Fleetwood, Y. V. White, R. Pasternak, S. N. Rashkeev, F. Brunier, N. Bresson, M. Fouillat, S. Cristoloveanu, and N. H. Tolk, IEEE Transactions on Nuclear Science, 51(6), (2004).

28. M. L. Alles, R. Pasternak, X. Lu, N. H. Tolk, R. D. Schrimpf, D. M. Fleetwood, R. P. Dolan, and R. W. Standley, Semiconductor Manufacturing, IEEE Transactions on, 20(2), (2007).

29. D. Damianos, G. Vitrant, M. Lei, J. Changala, A. Kaminski-Cachopo, D. BlancPelissier, S. Cristoloveanu, and I. Ionica, Solid-State Electronics, 143, (2018). 